\title{
Atomistic simulation on size-dependent yield strength and defects evolution of metal nanowires
}

\author{
Zhenyu Yang ${ }^{\mathrm{a}}$, Zixing Lu ${ }^{\mathrm{a}}$, Ya-Pu Zhao ${ }^{\mathrm{b}, *}$ \\ a School of Aeronautical Science and Engineering, Beihang University, Beijing 100191, PR China \\ ${ }^{\mathrm{b}}$ State Key Laboratory of Nonlinear Mechanics, Institute of Mechanics, Chinese Academy of Sciences, 15 Beisihuanxi Road, Beijing 100190, PR China
}

\section{A R T I C L E I N F O}

\section{Article history:}

Received 10 January 2009

Accepted 10 February 2009

Available online 19 March 2009

\section{PACS:}

61.46. $-\mathrm{W}$

62.20. $-\mathrm{X}$

Keywords:

Molecular dynamics (MD)

Modified embedded atom method (MEAM)

Nanowires

Size-dependent

Yield strength

\begin{abstract}
A B S T R A C T
A simple derivation based on continuum mechanics is given, which shows the surface stress is critical for yield strength at ultra-small scales. Molecular dynamics (MD) simulations with modified embedded atom method (MEAM) are employed to investigate the mechanical behaviors of single-crystalline metal nanowires under tensile loading. The calculated yield strengths increasing with the decrease of the cross-sectional area of the nanowires are in accordance with the theoretical prediction. Reorientation induced by stacking faults is observed at the nanowire edge. In addition, the mechanism of yielding is discussed in details based on the snapshots of defects evolution. The nanowires in different crystallographic orientations behave differently in stretching deformation. This study on the plastic properties of metal nanowires will be helpful to further understanding of the mechanical properties of nanomaterials.
\end{abstract}

(c) 2009 Elsevier B.V. All rights reserved.

\section{Introduction}

Nanowire has a sizable surface area to volume ratio as compared to bulk materials, which leads its mechanical properties to be quite different from those of bulk materials [1-3]. Metal nanowires, in particular, have attracted extensive attention in recent years because they have broad applications in many areas, such as nano-mechanical and nano-electronic devices [4], nanoscale integrated circuits [5], opto-electronic applications [6]. Besides the important applications in nanoelectromechanical systems (NEMS), the nanowires can also be used as tips for scanning tunneling microscope (STM) and atomic force microscope (AFM) [7]. The mechanical behaviors of nanostructures have been known to be subject to surface effects [8-11], which are closely related to size effects. Investigation on the mechanical properties is one of the key challenges that we need to overcome for the future technological applications of nanomaterials. As the scale is reduced to nanometer, the surface effects dramatically change the physical phenomena [10-12] and the yield strengths of the low-dimensional nanomaterials are different from the bulk materials [1318 ]. Although, many efforts have been devoted to elucidate size effects in crystalline nanostructures $[8,15,19]$, the size-dependent

\footnotetext{
* Corresponding author.

E-mail address: yzhao@imech.ac.cn (Y.-P. Zhao).
}

mechanical properties of nanostructure materials still need to be investigated further.

Atomistic simulations have been widely used to study a variety of deformation mechanisms in nanometer-scale materials [1014,20-26]. Tension-compression asymmetry [13], effects of temperature [16], size and strain rate effects [25] have been revealed as interesting phenomena. Gall et al. [20] predicted increasing nanowire strength with decreasing dimensional scale. In addition, the yield and fracture properties of the gold nanowires were investigated by molecular dynamics (MD) modeling using embedded atom method (EAM) [22]. Besides, the yield strength of nanowires was quantitatively predicted by MD simulations $[22,23,25,26]$. The previous experiences suggested that the accurate modeling of stacking fault and surface energies is critical in capturing the fundamental deformation behaviors of nanowires.

Although, various researches have uncovered interesting phenomena regarding the plastic deformation of nanomaterials, a comprehensive understanding of the observed behaviors is lacking. In this research, the yield condition of the nanowires was analyzed by a simple continuum model with the surface stress considered. von Mises yield condition was used to ascertain the value of the effective stress at initial yield point. The size-dependent yield stresses of single-crystalline metal nanowires were simulated by the atomistic simulations using modified embedded atom method (MEAM). The calculated results were followed by a particular 
discussion on yield mechanism of nanowire from the viewpoint of dislocation moving. A deep understanding to the plasticity of the nanowires under tensile loading was achieved.

\section{Effective yield stress}

Based on thermodynamics, surface free energy $\gamma$ is required to create a unit area of new free surface. The surface energy and surface stress tensor $\tau_{\alpha \beta}$ are connected by the Shuttleworth relationship

$\tau_{\alpha \beta}=\gamma \delta_{\alpha \beta}+\frac{\partial \gamma}{\partial \varepsilon_{\alpha \beta}}$

where $\delta_{\alpha \beta}$ is the Kronecker delta and $\varepsilon_{\alpha \beta}$ is the surface strain tensor. In the following analysis, the surfaces of the nanowires were assumed to be isotropic [15].

The effect of surface stress on the structure of nanowire can be appreciated by examining the magnitude of the intrinsic compressive stress-induced by the tensile surface stress (Fig. 1). For a nanowire, tensile surface stress existing on the surfaces can induce intrinsic compressive stresses in the interior of the nanowire $[13,14]$. The surface stresses for three group of side surfaces are represented by $\tau_{1}, \tau_{2}$ and $\tau_{3}$. At equilibrium, the tensile surface stresses and the intrinsic compressive stresses balance each other. Consequently, the stress state in the interior of the nanowire can be obtained as $\sigma_{x}^{i}=-\frac{2\left(b \tau_{3}+L \tau_{2}\right)}{b L}, \sigma_{y}^{i}=-\frac{2\left(a \tau_{3}+L \tau_{1}\right)}{a L}$ and $\sigma_{z}^{i}=-\frac{2\left(a \tau_{2}+b \tau_{1}\right)}{a b}$. Then the nanowire is subjected to the principal stresses

$\left\{\begin{array}{l}\sigma_{1}=-\frac{2\left(b \tau_{3}+L \tau_{2}\right)}{b L} \\ \sigma_{2}=-\frac{2\left(a \tau_{3}+L \tau_{1}\right)}{a L} \\ \sigma_{3}=\sigma_{e}-\frac{2\left(a \tau_{2}+b \tau_{1}\right)}{a b}\end{array}\right.$,

in which, $\sigma_{e}$ is for the effective yield stress. With the von Mises yield condition, the yield stress is expressed by

$\sigma_{y}=\frac{\sqrt{2}}{2} \sqrt{\left(\sigma_{1}-\sigma_{2}\right)^{2}+\left(\sigma_{2}-\sigma_{3}\right)^{2}+\left(\sigma_{3}-\sigma_{1}\right)^{2}}$.

Substituting the principle stresses in the yield condition, the effective yield stress can be obtained as

$\sigma_{e}=-\frac{A}{2} \pm \frac{1}{2} \sqrt{A^{2}-4 B+4 \sigma_{y}^{2}}$,

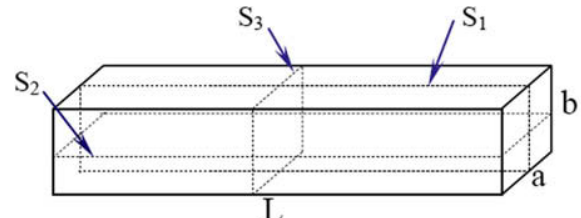

(a)

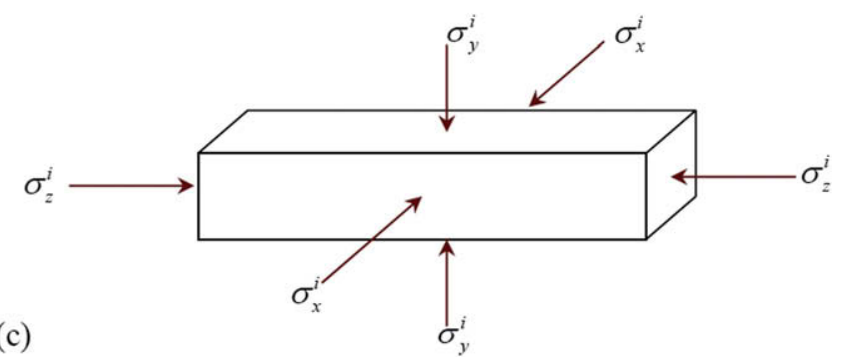

Fig. 1. Surface-stress-induced compressive stress in nanowire interior. (a) Nanowire with dimension of $a \times b \times L$. Three slices of $\mathrm{S}_{1}-\mathrm{S}_{3}$ are added to give an insight to the interior stress. (b) The coordinate system for the nanowire. (c) Stress state of the interior of the nanowire induced by surface stress. in which,

$A=\left(\frac{2}{a}-\frac{4}{b}\right) \tau_{1}+\left(\frac{2}{b}-\frac{4}{a}\right) \tau_{2}+\frac{4}{L} \tau_{3}$,

and

$$
\begin{aligned}
B= & 2\left[\frac{1}{a^{2}}+\frac{1}{b^{2}}+\left(\frac{1}{a}-\frac{1}{b}\right)^{2}\right]\left(\tau_{1}^{2}+\tau_{2}^{2}\right)+\frac{4}{L^{2}} \tau_{3}^{2}-\frac{4}{a b} \tau_{1} \tau_{2} \\
& -\frac{4}{a L} \tau_{1} \tau_{3}-\frac{4}{b L} \tau_{2} \tau_{3} .
\end{aligned}
$$

For a infinitely long nanowire with square cross-sectional area, $a=b, L \gg a$. So the Eqs. (5) and (6) can be approximately simplified to $A=-\frac{2}{a}\left(\tau_{1}+\tau_{2}\right)$ and $B=\frac{4}{a^{2}}\left(\tau_{1}^{2}+\tau_{2}^{2}\right)-\frac{4}{a^{2}} \tau_{1} \tau_{2}$, respectively, with the terms related to $1 / L$ being neglected reasonably. So the effective yield stress for a nanowire is

$\sigma_{e}=\frac{\tau_{1}+\tau_{2}}{a} \pm \sqrt{\sigma_{y}^{2}-\frac{3}{a^{2}}\left(\tau_{1}-\tau_{2}\right)^{2}}$

This is a general description for the yield stress of the nanowires, which is long enough. For the surface $\{100\}$ of face centered cubic (FCC) metal nanowire, $\tau_{1}$ is equal to $\tau_{2}$, but not for surface $<110>$ or surface $<111>$. For the sake of simplicity, $\tau_{1}$ is assumed to be equal to $\tau_{2}$, so a more succinct expression is obtained as $\sigma_{e}=\frac{2}{a} \tau_{1} \pm \sigma_{y}$. A graphical rendition of this formula by a plot of the dimensionless scale is shown in Fig. 2. The value of $\tau_{1}$ is set to be about $1 \mathrm{~J} / \mathrm{m}^{2}$, which is on the order of the surface stress of FCC metal surfaces. For a nanowire with lateral dimension $a \sim 1$ $\mathrm{nm}$, the contribution to the yield stress by surface stress can be estimated as about $2 \mathrm{GPa}$. The magnitude of $\tau_{1}$ in nanowires is significant, on the order of GPa, and can potentially drive numerous stress-induced phenomena $[10,12,27]$. In fact, the surface stress is changing with area of the surfaces being stretched, but the effect of surface stretching is not considered here for simplicity. This result also indicates that the yield of the nanowire is asymmetric, and shows the magnitude of the tensile yield stress is larger than that of the compressive yield stress for small nanowires $[9,13]$. For $a \sigma_{y} / \tau_{1}<2$, the nanowire yields under its own surface stressinduced intrinsic compressive stress and no external force at all is necessary.

Eq. (4) can also be extended to other low-dimensional nanostructures. For a ultra-thin nanofilm, $a=L \gg b$. The effective yield stress for the nanofilm can be obtained as

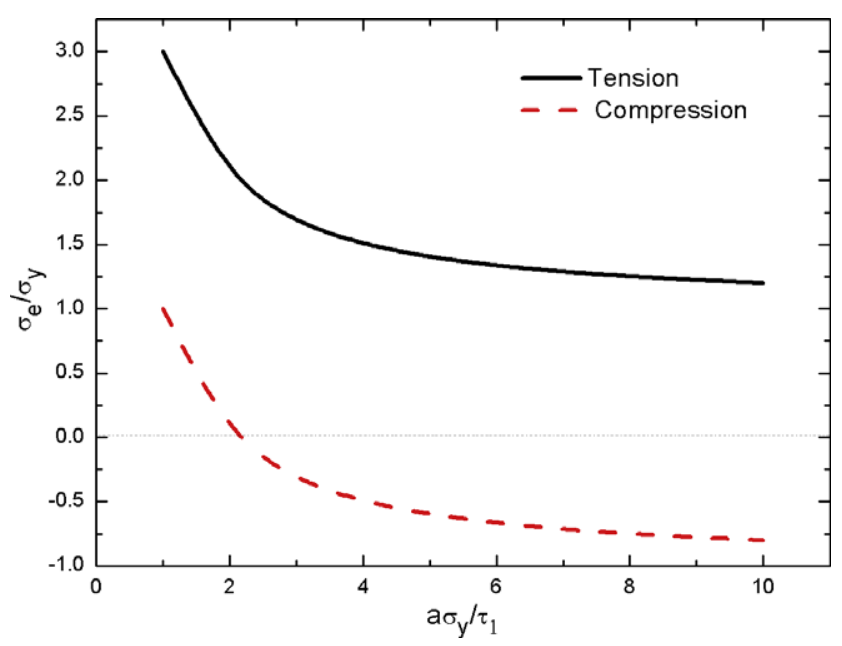

Fig. 2. A plot of the dimensionless effective yield stress versus the dimensionless width. The solid line is for the tension of the nanowire, and dashed line for the compression. 
$\sigma_{e}=\frac{\tau}{b} \pm \sqrt{\sigma_{y}^{2}-3\left(\frac{\tau}{b}\right)^{2}}$

which is reduced to the model of Gioia and Dai [15].

\section{Molecular dynamics simulation}

The MD simulation was carried out using the MEAM potential developed by Baskes [28], which has been successfully used in describing phase transformation [10] and elastic properties prediction of nanowires [21]. It incorporates the angular forces that result from the directional bonding by modifying the background electron density from the spherically averaged density used in EAM [29]. In both the EAM and MEAM, the total energy of a system of atoms can be expressed as

$U=\sum_{i}^{N}\left(F_{i}\left(\bar{\rho}_{i}\right)+\frac{1}{2} \sum_{j \neq i}^{N} \phi_{i j}\left(r_{i j}\right)\right)$,

where $F_{i}\left(\bar{\rho}_{i}\right)$ is the embedding energy required to place atom $i$ in a local electron density $\bar{\rho}_{i}$, and $\phi_{i j}$ is the two-body potential between atoms $i$ and $j$, and $r_{i j}$ is the distance between atoms $i$ and $j$. The summation above is over the total number of atoms $N$ in the system. In the EAM, $\bar{\rho}_{i}$ is given by a linear supposition of spherically averaged atomic electron densities, while in the MEAM, $\bar{\rho}_{i}$ is augmented by an angularly dependent term [28]. With the angularly dependent term, MEAM can be used to model both metallic and covalent materials. The parameters used in this simulation were taken from Ref. [28] and were fitted to the FCC lattice constant, the bulk modulus, structural energy, and the vacancy formation energy.

Nanowires with different crystallographic orientations were created out of a bulk FCC metal crystal. The nanowires are all 40 cubic lattice units (CLUs) long at the $Z$ direction (Fig. 3), with a square cross-section and the dimension varying from 6 CLUs to 10 CLUs. The tensile deformation of $\langle 100>/\{100\}$ metal nanowire was not considered here, which has been frequently studied in the reported literatures $[14,20,23]$. For $\langle 110>$ and $<111>$ nanowires, the cross-sections were made to be as square as possible. In order to obtain equilibrium configurations, the nanowires were relaxed to an equilibrium minimum energy configuration with all free boundary conditions and then thermally equilibrated to $300 \mathrm{~K}$ for 50 ps using the Nose-Hoover thermostat [30] with a time step of $2 \mathrm{fs}$, keeping the length of the wires constant. Approximation to quasi-static tensile loading in each deformation increment was achieved in two steps [31]. The loading was applied along the axis of the nanowires, with the bottom layers fixed, while the rest atoms were displaced in accordance with a prescribed uniform strain in the length direction, and then the wires were dynamically relaxed for 20 ps to obtain the microscopic equilibrium configuration. The average stress over the last 5 ps of the relaxation period was taken as the stress of the nanowires. The velocity Verlet algorithm [32] was used to integrate the equations of motion and all MD simulations in this study were performed using the parallelized code LAMMPS [33]. The snapshots of the MD results were processed by Atomeye [34].
The average stresses in the atomistic systems were calculated using the virial theorem $[35,36]$, which takes the form

$\sigma^{\alpha \beta}=\frac{1}{\Omega}\left[-\sum_{i} m_{i} v_{i}^{\alpha} v_{i}^{\beta}+\frac{1}{2} \sum_{i} \sum_{j \neq i} F_{i j}^{\alpha} \beta_{i j}^{\beta}\right]$,

where the summation is over all the atoms occupying the total volume $\Omega$, and the first term is a kinetic energy component for atom $i$. $m_{i}$ and $v_{i}$ are the mass and velocity of atom $i, F_{i j}$ is the force between atom $i$ and $j$, and the indices $\alpha$ and $\beta$ denote the Cartesian components. $r_{i j}^{\alpha}$ is the projection of the inter-atomic distance vector along coordinate $\alpha$. The second summation term in the right side of Eq. (10) is due to the inter-atomic force.

\section{Results and discussions}

This study addresses tensile tests of $\mathrm{Ni}$ and $\mathrm{Cu}$ nanowires with $<110>$ and <111> crystallographic orientations, respectively. Stress-strain relationships are obtained to locate the initial yield point and to determine the size effect on yield stress. The snapshots of the tensile deformation are captured to understand the yield mechanisms of the nanowires under uniaxial tensile loading. In order to examine the details in its interior structure, deformations of the nanowire shape and inside defects evolution are snapped from different perspectives.

The tensile stress-strain responses for $\mathrm{Ni}<110\rangle$ nanowires are shown in Fig. 4. The yield stress increases with the decrease of the cross-section size. The $\mathrm{Ni}<110>$ nanowire with lateral dimension of $2.1 \mathrm{~nm}$ yields at the stress of about $14.26 \mathrm{GPa}$, and the yield stress falls to $12.29 \mathrm{GPa}$ for the nanowire with width of $3.5 \mathrm{~nm}$. Snapshots of the defects are shown in Fig. 5 for $\mathrm{Ni}<110>$ nanowire

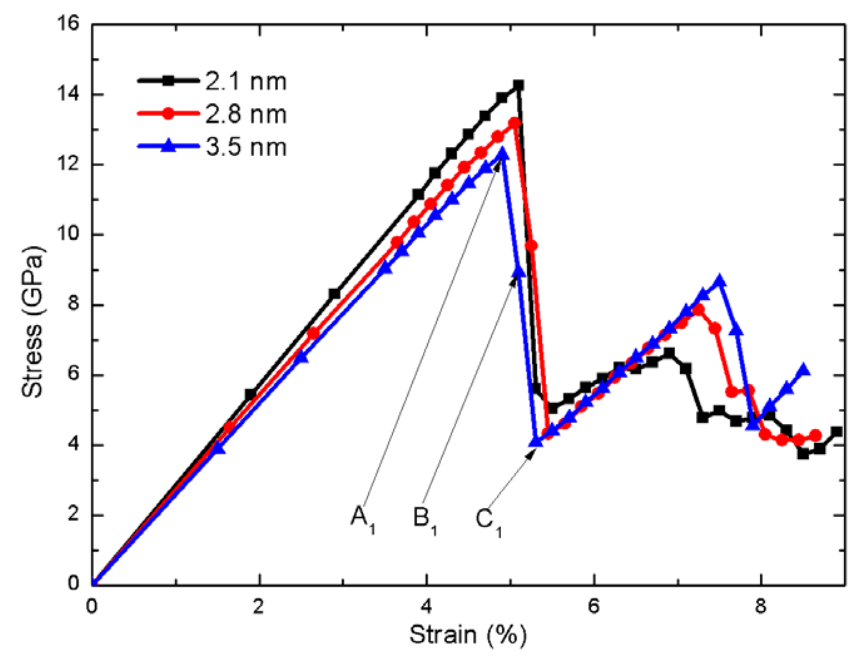

Fig. 4. Stress-strain curves for $\mathrm{Ni}<110>$ nanowires as a function of lateral dimension. The snapshots of the deformations at the point $A_{1}-C_{1}$ are shown in following Fig. 5a-c, respectively.

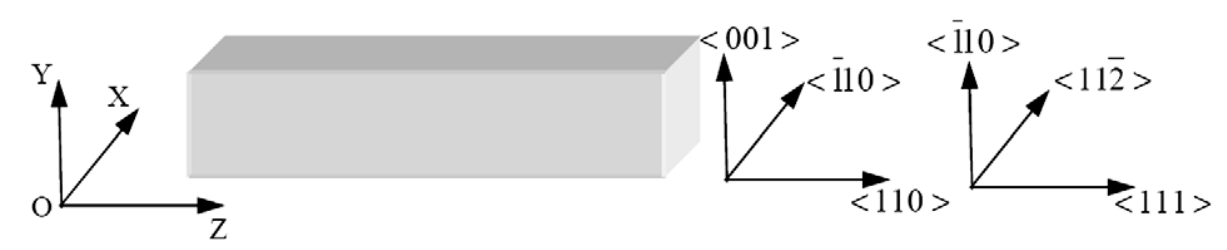

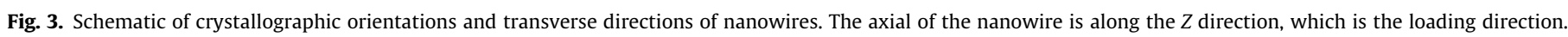




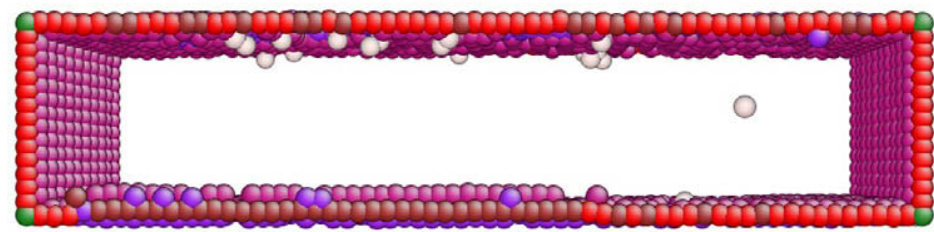

(a)

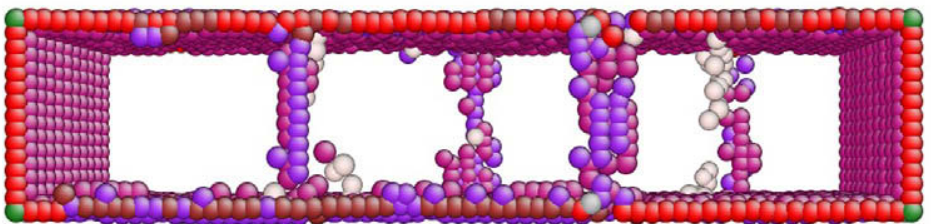

(b)

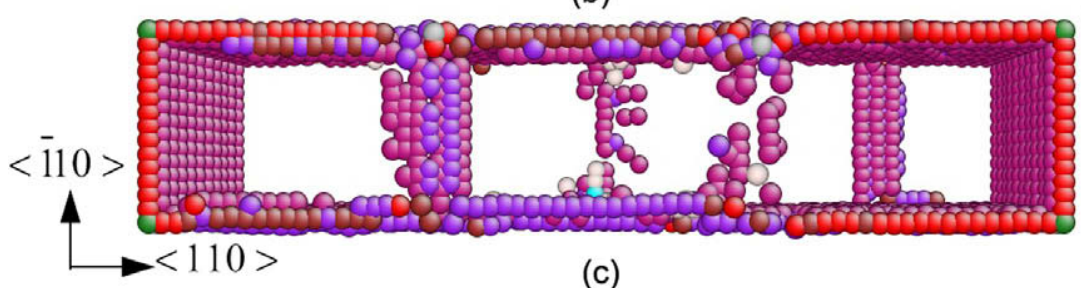

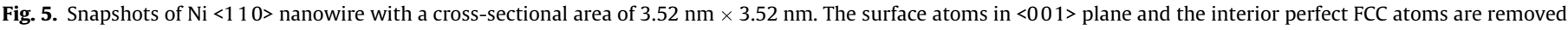

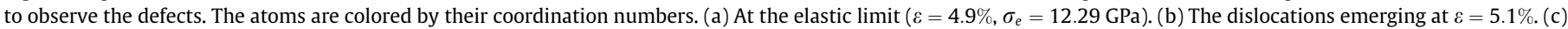
Strain at $5.3 \%$.

with a cross-sectional area of $3.52 \mathrm{~nm} \times 3.52 \mathrm{~nm}$, with the atom colored by coordination number [34]. The points $A_{1}-C_{1}$ in Fig. 4 are related to the figures a-c in Fig. 5, respectively. Fig. 6 shows the deformation evolution of the Ni nanowire under tensile loading. Fig. 5a is the snapshot the elastic limit, with the nucleation at the surfaces. At the point $\mathrm{B}_{1}$, two parallel stacking faults result in surface steps on surfaces $\{001\}$ and can be observed in Figs. 5b and 6a. In Fig. 5c, a new emerging stacking fault shows at strain of 5.3\%. After the point $C_{1}$, the elastic strain builds up stress until next yielding, which is also found in compression loading of $\mathrm{Ni}$ $<111>$ nanowires [31]. This can be reasonably attributed to dislocation starvation, which is led by dislocations propagating across the wire cross-section and then moving out of the wires. These snapshots indicate that slip is a dominating factor in the plastic

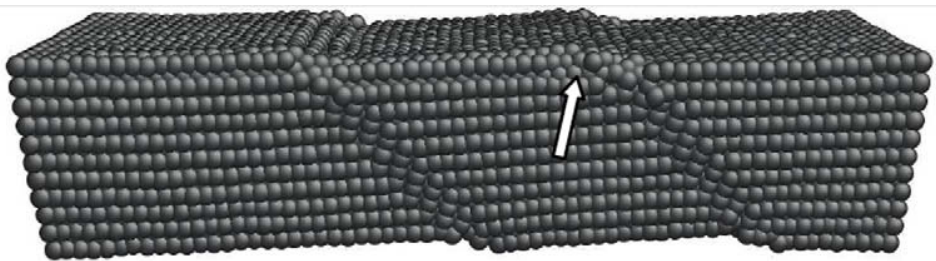

(a)

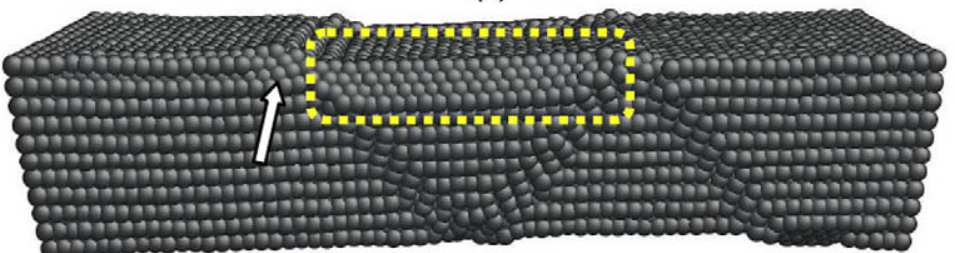

(b)

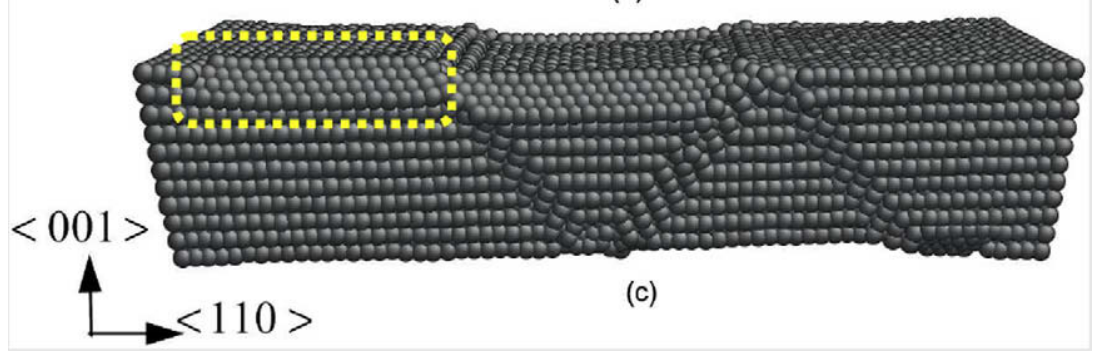

Fig. 6. Deformation of $\langle 110\rangle$ single-crystalline Ni nanowire under tension. (a) Strain at $5.1 \%$. (b) Strain at $5.3 \%$. (c) Strain at $8.5 \%$. The arrows point at the initially reoriented place and the reorientation is observed in the dashed frame. 


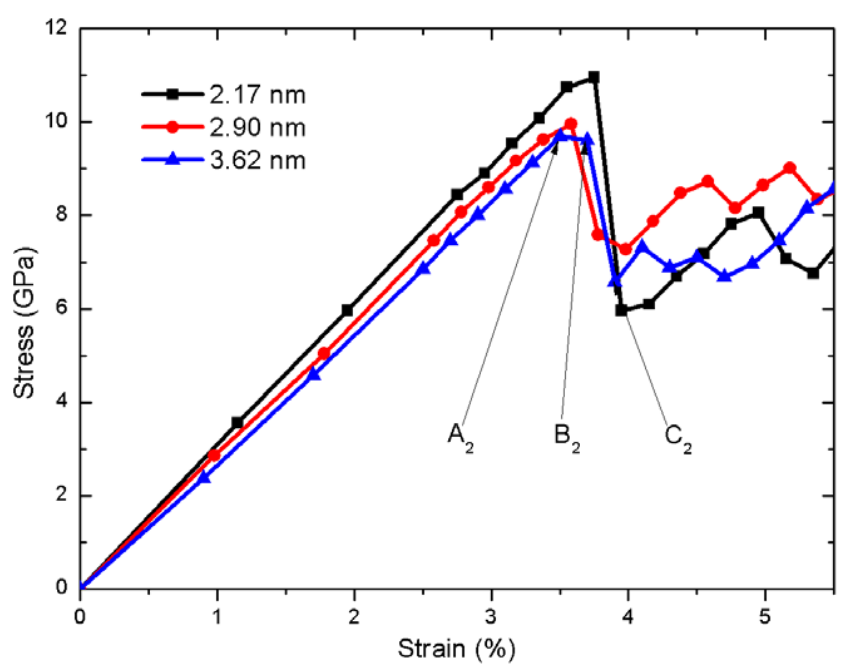

Fig. 7. Stress-strain curves for $\mathrm{Cu}<110>$ nanowire as a function of lateral dimension. The snapshots of the deformations at the point $A_{2}-C_{2}$ are shown in Fig. 8a-c, respectively.

deformation, which is evident by the stacking faults observed along $\{111\}$ plane. The analogous phenomenon was observed in MD simulation using EAM [22]. Surface reorientation between the two parallel stacking faults is observed at the edge of the two side surfaces (Fig. 6b). The generated surface after reorientation is proved to be a $\{111\}$ surface. A newly reoriented surface,

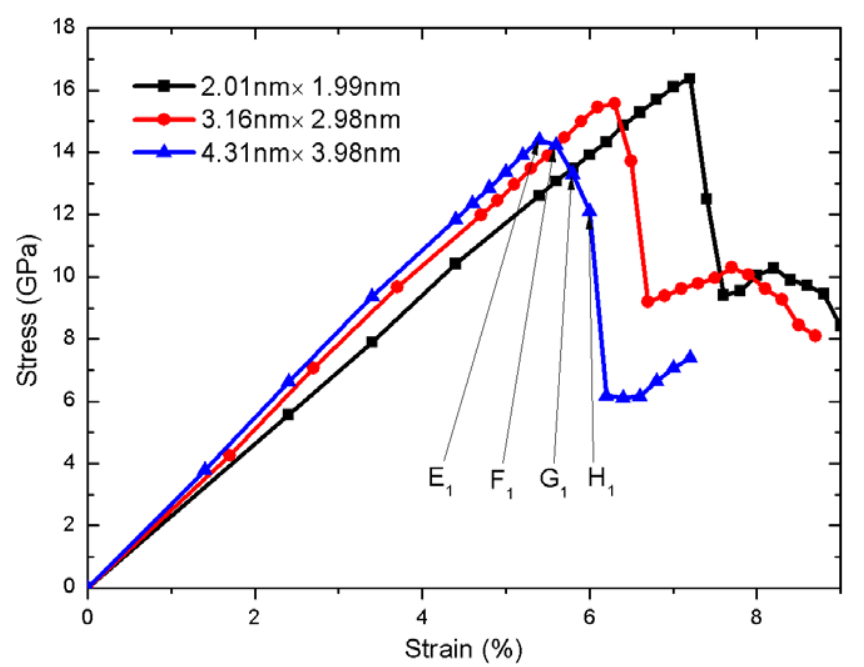

Fig. 9. Stress-strain relationship for $\mathrm{Ni}<111>$ nanowires under tensile loading as functions of cross-sectional area. Snapshots of the four tagged points are shown in Fig. 10.

which originates from the arrow-pointed place in Fig. $6 \mathrm{~b}$ and also locates on the edge of the wire, shows at strain of $8.5 \%$ (Fig. 6c).

The stress-strain responses for $\mathrm{Cu}<110>$ nanowires loaded in tension are shown in Fig. 7. The result indicates that the smaller wire yields at higher stress. The snapshots of defects at points $\mathrm{A}_{2}-\mathrm{C}_{2}$ are shown in Fig. 8. For $\mathrm{Cu}<110>$ nanowire, the mechanism

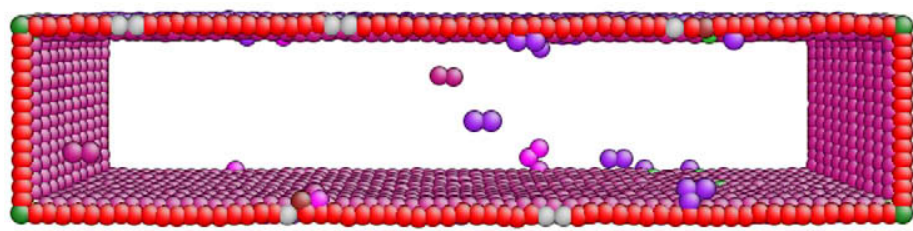

(a)

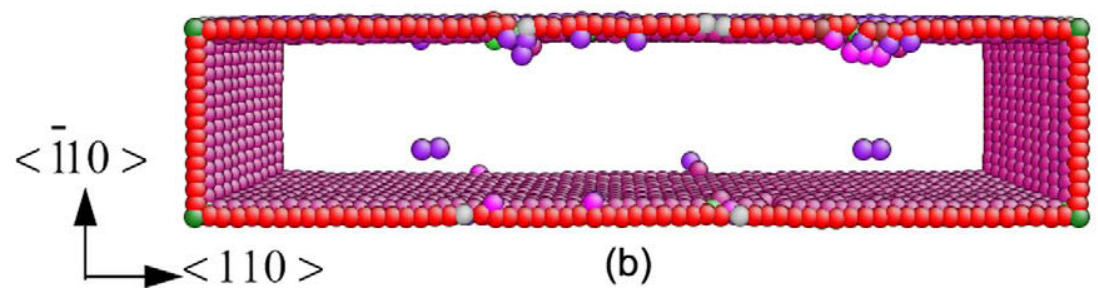

(b)
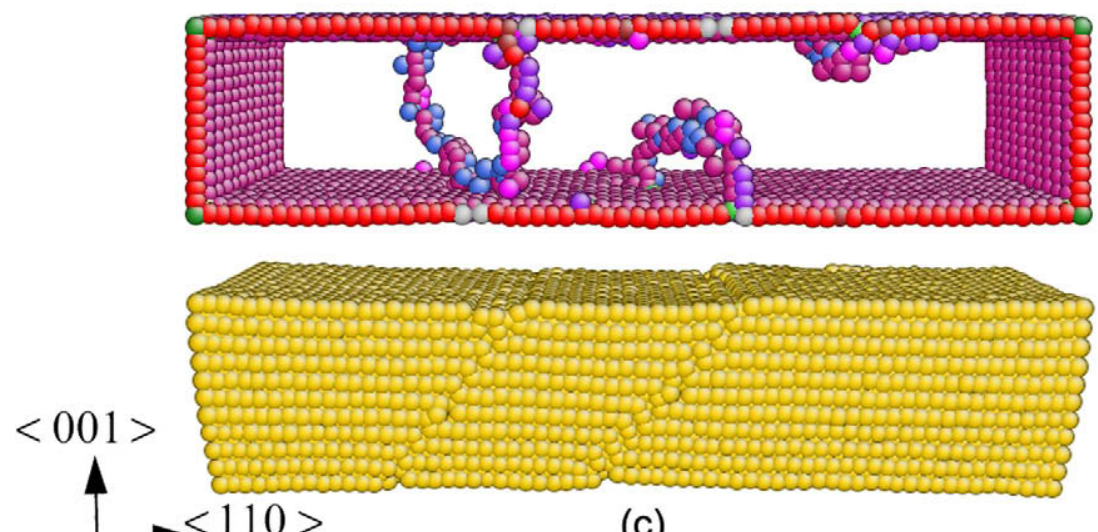

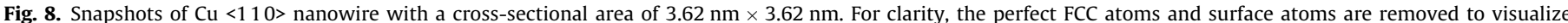

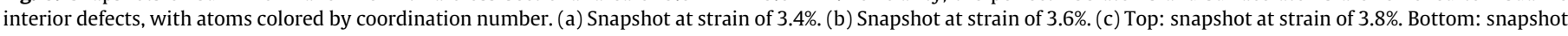
of parallel stacking fault structure at the same strain level 


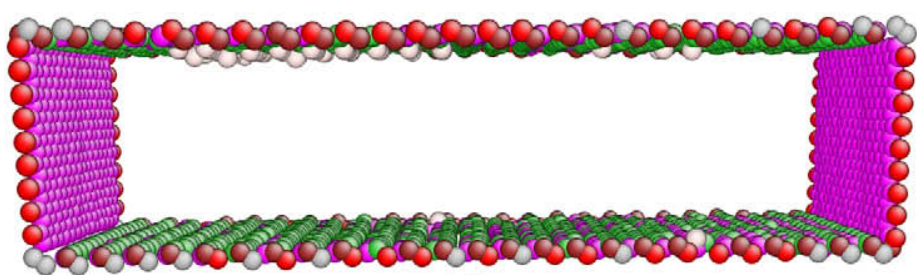

(a)

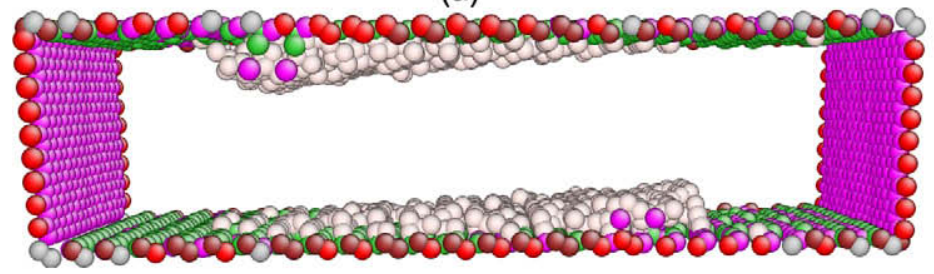

(b)

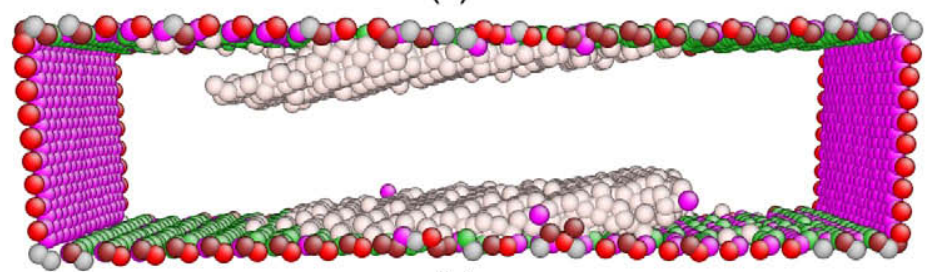

(c)

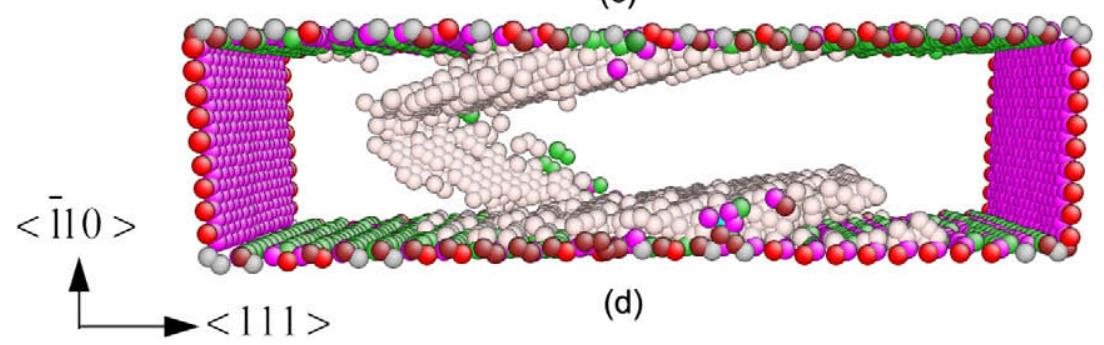

Fig. 10. Snapshots of the $\mathrm{Ni}<111>$ nanowire with a cross-sectional area of $4.31 \mathrm{~nm} \times 3.98 \mathrm{~nm}$. For clarity, only the defective atoms and side surfaces are displayed, each atom is colored according to the coordination number. (a) Snapshot at strain of 5.4\%, with $\sigma_{e}=14.39 \mathrm{GPa}$. (b) Snapshot at strain of 5.6\%. (c) Snapshot at strain of 5.8\%. (d) Snapshot at strain of $6.0 \%$

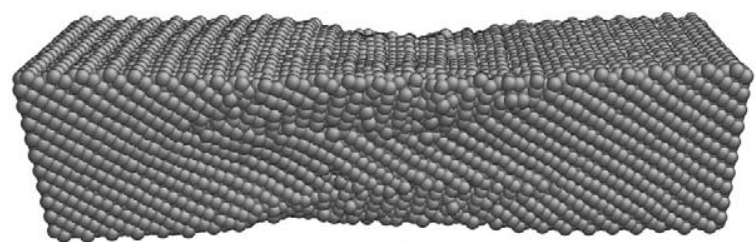

(a)

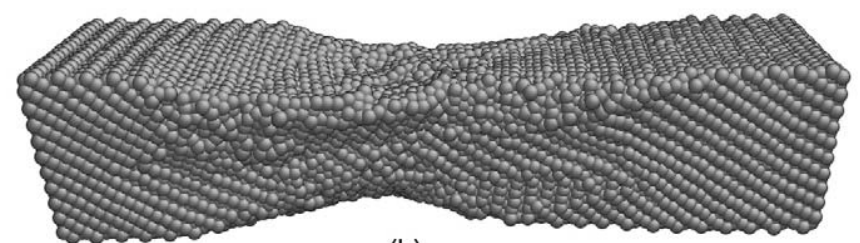

(b)

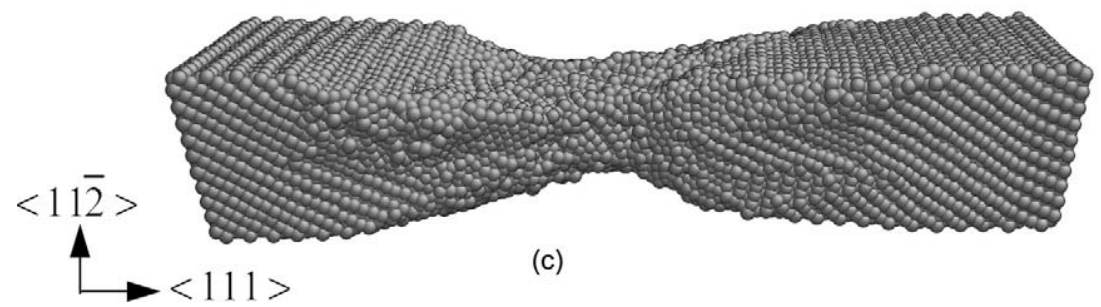

Fig. 11. Snapshots of shape deformation of $\mathrm{Ni}<111>$ nanowire under tensile loading. (a) Strain at $10 \%$. (b) Strain at $25 \%$. (c) Strain at $41 \%$. 


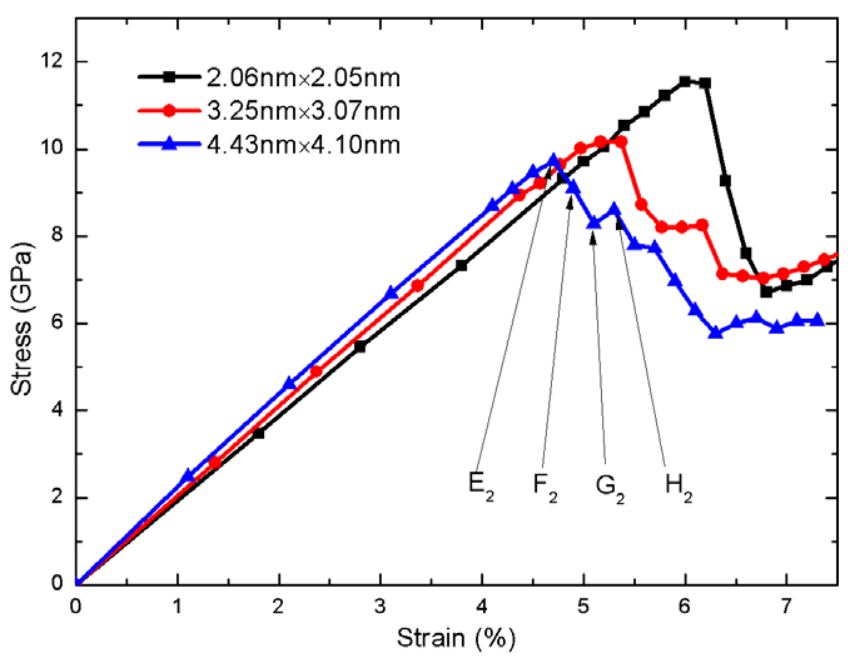

Fig. 12. Stress-strain curves for $\mathrm{Cu}<111>$ nanowire as functions of cross-sectional area. Snapshots of the four tagged points are shown in Fig. 13.

of yield illustrated in Fig. 8 is similar to $\mathrm{Ni}<110\rangle$ nanowire. Nucleation of dislocations at the surface is found at the elastic limit (Fig. 8a). Fig. 8b is the snapshot before the dislocation emission, which is corresponding to point $\mathrm{B}_{2}$ on the stress-strain curve. The dislocations nucleate at the bottom surface and emit to the opposite surface and the dislocations move along $<\overline{1} 10>$ direction. Two parallel stacking faults emerge at the strain of $3.8 \%$ (Fig. 8c), leading the stress-strain curves to a sharp decline. With the strain increase, the $\mathrm{Cu}<110>$ nanowire shows a new stacking fault between the two existing parallel stacking faults. Reorientation from the edge of surface $\{001\}$ and surface $\{\overline{1} 10\}$ to surface $\{111\}$ can also be observed.

The $<111>$ nanowires with two $\{11 \overline{2}\}$ and two $\{\overline{1} 10\}$ side surfaces are also tested by uniaxial tensile loading. The stress-strain relationships for $\mathrm{Ni}<111>$ nanowire are illustrated in Fig. 9 with lateral dimension varying from $2.0 \mathrm{~nm}$ to about $4.3 \mathrm{~nm}$. The tension test of $\langle 111\rangle$ nanowires also shows that the yield stress is size-dependent. The Ni $<111>$ nanowire with a cross-section area of $2.01 \mathrm{~nm} \times 1.99 \mathrm{~nm}$ yields at the stress of $16.38 \mathrm{GPa}$, which is larger than that of bigger nanowires. Four snapshots are given in Fig. 10 according to the four tagged points on the stress-strain curve in Fig. 9. As load increase, the defects begin to generate at both of the $\langle\overline{1} 10>$ surfaces inside the wires, which can be observed obviously at elastic limit (Fig. 10a). The defects continue to grow in snapshots of Figs. $10 \mathrm{~b}$ and $\mathrm{c}$. At the point $\mathrm{H}_{1}$ on the stress-strain curve in Fig. 9, the defects at the top and the bottom finally joint together at the strain of $6.0 \%$ (Fig. 10d). There is a sharp fall of stress after point $\mathrm{H}_{1}$, which indicates the junction of the

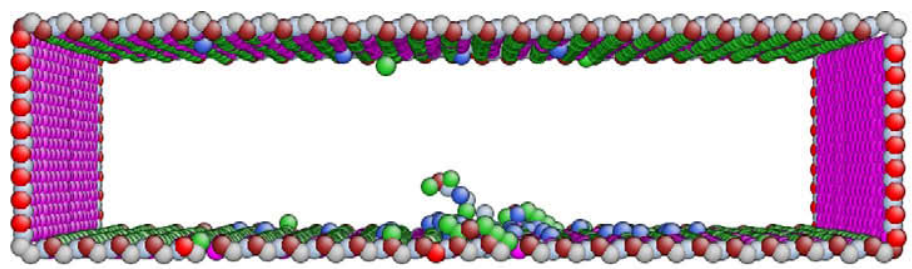

(a)

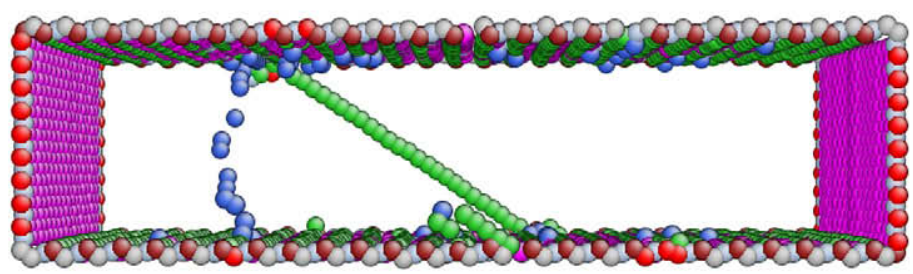

(b)

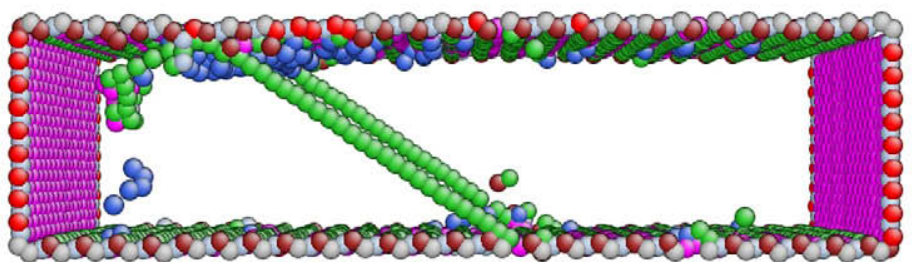

(c)

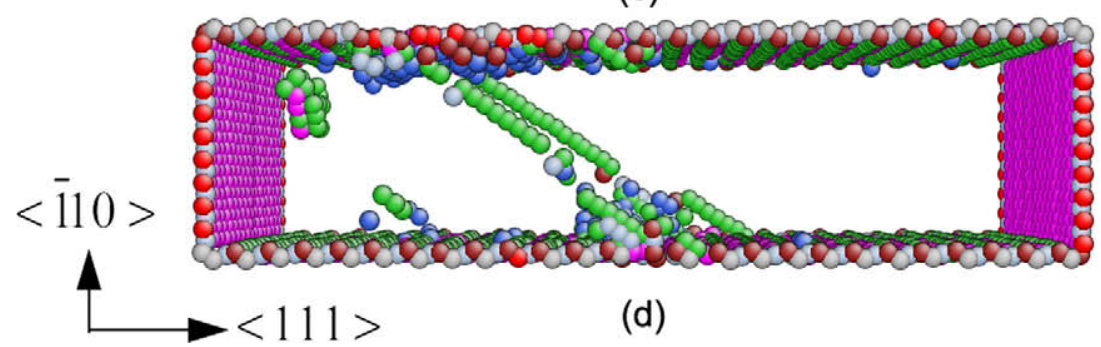

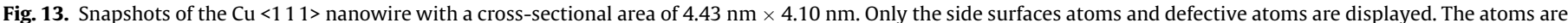

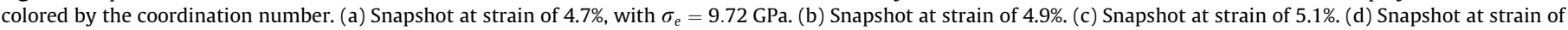
$5.3 \%$. 
defects makes the nanowire failed and leads to a sudden decline of stress. The snapshots of the shape evolution are shown in Fig. 11. As deformation increase, necking sets in and leads to failure.

The stress-strain responses for $\mathrm{Cu}<111>$ nanowires loaded in uniaxial tension are shown in Fig. 12. The yield strengths for the $\mathrm{Cu}<111>$ nanowires are also proved to be size-dependent. The $\mathrm{Cu}<111>$ nanowires are found to behave differently from $\mathrm{Ni}$ nanowires in tension deformation. Four snapshots of the $\mathrm{Cu}<111>$ nanowires deformation are shown in Fig. 13, which correspond to the four tagged points in Fig. 12. Unlike $\mathrm{Ni}<111>$ nanowires, $\mathrm{Cu}<111>$ nanowires show that the dislocations nucleate at the edges, and extend from the bottom $\{\overline{1} 10\}$ surface to the top $\{110\}$ surface with an angle of $45^{\circ}$ to the axial direction (Fig. 13a and c). Two parallel dislocations at the strain of $5.1 \%$ are on the same plane (Fig. 13c). With the strain increase, more dislocations are emerging (Fig. 13d) at the edges of the nanowires. These snapshots demonstrate that the dislocations are nucleated at surface edges and lead to a glide surface.

From the stress-strain relationships in Figs. 4, 7, 9 and 12, the size-dependent elastic properties can also be concluded. For $\mathrm{Ni}$ $<110>$ and $\mathrm{Cu}<110>$ nanowires, the Young's moduli increase with the decrease of lateral dimension of wires. While, the $<111>$ nanowires show reverse characteristics that the Young's moduli decrease with the decrease of the lateral dimension of wires. The size-dependent elastic properties can be understood from the viewpoint of surface relaxation [8]. For concision, the yield stresses in the stress-strain curves of tensile tests are extracted out and illustrated in Fig. 14. The stress at the initial yield points on the stress-strain curve are defined as the yield strengths of the nanowires. The results of MD simulation on the tensile deformation show that $\mathrm{Ni}$ nanowires have the higher yield strengths than $\mathrm{Cu}$ nanowires. The yield strengths of the nanowires increase with the decrease of the lateral dimension. The regularity of change of yield strengths is consistent with the foregoing theoretical analysis, qualitatively. Based on the Eq. (7), the surface stress contributes to the effective yield strength, which is inversely proportional to the lateral dimension of the nanowires. This is also demonstrated by previous MD simulations $[13,20]$ and theoretical investigation [9]. With the cross-sectional dimension decreases, the surface area to volume ratio increases and the surface stressinduced internal stress becomes considerable, which leads to the size-dependent mechanical properties.

The data in Table 1 indicate that surface $\{111\}$ has the lowest surface energy between the typical surfaces. Surfaces $\{100\}$ and $\{110\}$ are shown to change into $\{111\}$ surface under tensile load-

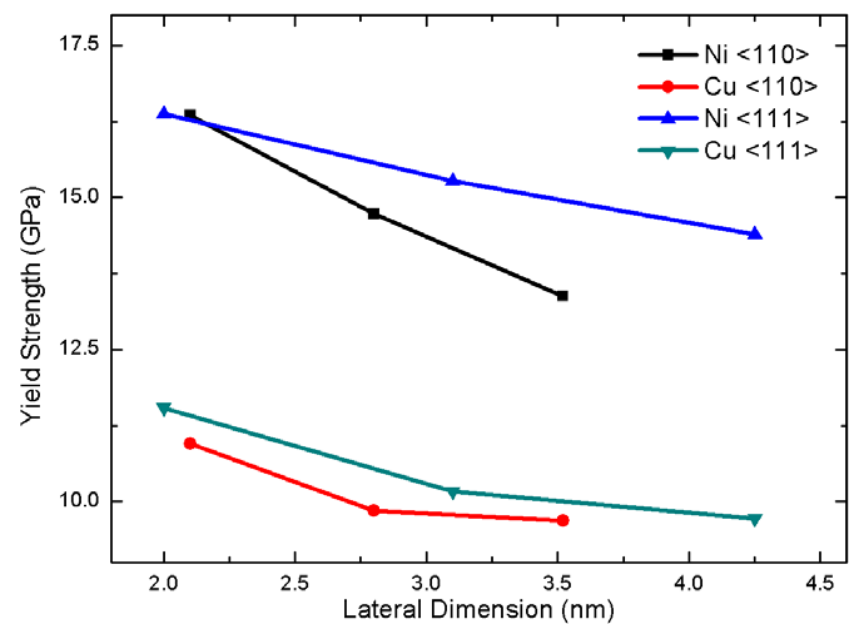

Fig. 14. The yield strengths for the nanowries as functions of lateral dimension.
Table 1

Comparisons of the surface energy of $\mathrm{Ni}$ and $\mathrm{Cu}$ estimated by EAM and MEAM. Surface energies are all in $\mathrm{J} / \mathrm{m}^{2}$.

\begin{tabular}{llll}
\hline Materials & $<100\rangle$ & $\langle 110\rangle$ & $<111\rangle$ \\
\hline Ni (EAM) [37] & 1.59 & 1.73 & 1.45 \\
Ni (MEAM) [29] & 2.42 & 2.37 & 2.02 \\
Cu (EAM) [22] & 1.35 & 1.48 & 1.17 \\
Cu (MEAM) [29] & 1.65 & 1.61 & 1.41 \\
\hline
\end{tabular}

ing of certain orientations. The reorientation of the higher energy surfaces to $\{111\}$ surface is observed in the tension test of both $\mathrm{Ni}<110>$ and $\mathrm{Cu}<110>$ nanowires. Tension of $<110>$ nanowires verify the formation of stacking faults, which lead to plastic deformation. But no stacking fault is observed in tension of $<111>$ nanowires. The yield strengths of $\mathrm{Ni}<110>$ and $\mathrm{Cu}<110>$ nanowires are higher than those estimated by EAM [22], which can be attributed to that MEAM estimates higher surface energy than EAM does. The surfaces play an important role in the mechanical behavior of nanowires. The surface effects lead to intrinsic compressive stresses and dislocations resistant wire structures [13], both of which attribute to increase in tensile yield strength.

\section{Conclusions}

Based on continuum mechanics, the size-dependent yield properties of the nanowires are analyzed. The yield strength of the nanowires is found to be inversely proportional to the lateral dimension of the nanowires. Due to the effect of tensile surface stresses, nanowires can exhibit yield strength asymmetry.

MD simulations are employed to analyze the deformation behaviors and the yield mechanisms of FCC metal nanowires. The stress-strain relationships and yield strengths are obtained systematically. Both of the elastic properties and plastic properties are found to be size-dependent. The calculated yield strengths of the nanowires increase with the decrease of cross-sectional dimension. The results agree with the theoretical prediction, qualitatively. The analogously size-dependent mechanical properties are also revealed in other low-dimensional nanomaterials, such as $\mathrm{Cu}$ $<100>$ nanowires [26], Au nanowires [20] and certain semiconductor structures etc. The reorientation at the $\langle 110\rangle$ nanowire edges is observed, which is different from the phenomenon in MD simulation by EAM [22] because of the correction of angularly dependent term in the MEAM potential. For $<111>$ nanowires, Ni and $\mathrm{Cu}$ nanowires behave differently in defects generation and evolution. The effects of crystallographic orientation on the yield strength are also characterized.

\section{Acknowledgements}

ZYY was supported by the China Postdoctoral Science Foundation under the Grant No. 20080440013. ZXL was jointly supported by the National Natural Science Foundation of China (NSFC, Grant No. 10572013) and the Common Construction Project of Education Committee of Beijing under the Grant No.XK100060522. YPZ was supported by the NSFC (Grant Nos. 10772180 and 10721202), the National Basic Research Program of China (973 Program, Grant No. 2007CB310500) and the National High-tech R\&D Program of China (863 Program, Grant No. 2007AA04Z348).

\section{References}

[1] E. Tosatti, S. Prestipino, S. Kostlmeier, A. Dal Corso, F.D. Di Tolla, Science 291 (2001) 288.

[2] A.I. Yanson, I.K. Yanson, J.M. van Ruitenbeek, Phys. Rev. Lett. 84 (2000) 5832.

[3] G. Bilalbegovic, Comput. Mater. Sci. 18 (2000) 333. 
[4] J. Burki, C.A. Stafford, D.L. Stein, Phys. Rev. Lett. 95 (2005) 090601.

[5] S. Dubois, L. Piraux, J.M. George, K. Ounadjela, J.L. Duvail, A. Fert, Phys. Rev. B 60 (1999) 477.

[6] A. Christ, T. Zentgraf, J. Kuhl, S.G. Tikhodeev, N.A. Gippius, H. Giessen, Phys. Rev. B 70 (2004) 125113.

[7] N. Agraït, G. Rubio, S. Vieira, Phys. Rev. Lett. 74 (1995) 3995.

[8] J.G. Guo, Y.P. Zhao, J. Appl. Phys. 98 (2005) 074306

[9] W.X. Zhang, T.J. Wang, X. Chen, J. Appl. Phys. 103 (2008) 123527.

[10] J.K. Diao, K. Gall, M.L. Dunn, Nat. Mater. 2 (2003) 656

[11] J. Diao, K. Gall, M.L. Dunn, Phys. Rev. B 70 (2004) 075413

[12] W.W. Liang, D.J. Srolovitz, M. Zhou, J. Mech. Phys. Solids 55 (2007) 1729.

[13] J.K. Diao, K. Gall, M.L. Dunn, Nano Lett. 4 (2004) 1863.

[14] J.K. Diao, K. Gall, M.L. Dunn, J.A. Zimmerman, Acta Mater. 54 (2006) 643.

[15] G. Gioia, X. Dai, J. Appl. Mech.-Trans. ASME 73 (2006) 254.

[16] S.P. Ju J.S. Lin, WJ. Lee, Nanotechnology 15 (2004) 1221.

[17] B. Wu, A. Heidelberg, J.J. Boland, Nat. Mater. 4 (2005) 525.

[18] P.S. Branício, J.P. Rino, Phys. Rev. B 62 (2000) 16950.

[19] R.E. Miller, V.B. Shenoy, Nanotechnology 11 (2000) 139

[20] K. Gall, J.K. Diao, M.L. Dunn, Nano Lett. 4 (2004) 2431.

[21] J.K. Diao, K. Gall, M.L. Dunn, J. Mech. Phys. Solids 52 (2004) 1935.
[22] H.S. Park, K. Gall, J.A. Zimmerman, J. Mech. Phys. Solids 54 (2006) 1862.

[23] H.S. Park, J.A. Zimmerman, Phys. Rev. B 72 (2005) 054106.

[24] A.R. Setoodeh, H. Attariani, M. Khosrownejad, Comput. Mater. Sci. 44 (2008) 378.

[25] H. Ikeda, Y. Qi, T. Çagin, K. Samwer, W.L. Johnson, W.A. Goddard, Phys. Rev. Lett. 82 (1999) 2900.

[26] H.A. Wu, Mech. Res. Commun. 33 (2006) 9.

[27] H.S. Park, K. Gall, J.A. Zimmerman, Phys. Rev. Lett. 95 (2005) 255504.

[28] M.I. Baskes, Phys. Rev. B 46 (1992) 2727.

29] J. Wan, Y.L. Fan, D.W. Gong, S.G. Shen, X.Q. Fan, Model. Simul. Mater. Sci. 7 (1999) 189.

[30] S. Nose, J. Chem. Phys. 81 (1984) 5.

[31] A.J. Cao, E. Ma, Acta Mater. 56 (2008) 4816.

32] L. Verlet, Phys. Rev. 159 (1967) 98.

[33] S. Plimpton, J. Comput. Phys. 117 (1995) 1.

[34] J. Li, Model. Simul. Mater. Sci. 11 (2003) 173.

[35] K.S. Cheung, S. Yip, J. Appl. Phys. 70 (1991) 5688.

[36] M. Zhou, Philos. Trans. Roy. Soc. A - Math. Phys. 459 (2003) 2347.

[37] Z.Y. Yang, Y.P. Zhao, Surf. Rev. Lett. 14 (2007) 661. 